\section{Rickettsia honei Infection in Human, Nepal, 2009}

\section{Holly Murphy, ${ }^{1}$ Aurélie Renvoisé, ${ }^{1}$ Prativa Pandey, Philippe Parola, and Didier Raoult}

We report a case of Rickettsia honei infection in a human in Nepal. The patient had severe illness and many clinical features typical of Flinders Island spotted fever. Diagnosis was confirmed by indirect immunofluorescent assay with serum and molecular biological techniques. Flinders Island spotted fever may be an endemic rickettsiosis in Nepal.

$\mathrm{T}$ ick-borne rickettsioses are emerging zoonoses of marked endemicity caused by spotted fever group (SFG) rickettsia. Interest in rickettsioses is associated with increased description of new species and diseases, but this increase is unevenly distributed worldwide. Among patients with fever in Nepal, murine typhus and scrub typhus are frequently described (1), but tick-borne rickettsioses remain underinvestigated. Rickettsia honei is an SFG species that was described as a new species in 1998 and as the cause of Flinders Island spotted fever (FISF) in Australia (2,3). One human case of FISF has been confirmed in Thailand (4). We report a case of tick-borne rickettsiosis in Nepal caused by $R$. honei and highlight the necessity for heightened interest in emerging rickettsioses in Asia.

\section{The Patient}

A 67-year-old woman was admitted to the Canadian International Water and Energy Consultants Clinic Travel Medicine Center in Kathmandu, Nepal, in April 2009. She had a 5-day history of fever $\left(40.3^{\circ} \mathrm{C}\right)$, headache, diarrhea, and severe arthralgias. Results of a physical examination were unremarkable. Laboratory tests showed a leukocyte count of 6,500 cells $/ \mathrm{mm}^{3}$, an increase in immmature neutrophils and polymorphonuclear leukocytes, and thrombocytopenia. Treatment was initiated with intravenous ceftriaxone, $2 \mathrm{~g}$ every 24 h for 8 days, for suspected enteric fever.

Within 48 hours, her condition worsened. The patient had photosensitivity, tinnitus, frontal headache, insomnia, confusion, cough, distress, hypotension, tachycardia, hypoxia $\left(88 \%\right.$ oxygenation with $2 \mathrm{~L}$ of $\left.\mathrm{O}_{2}\right)$, and fever $\left(38.4^{\circ} \mathrm{C}\right)$. She was also disoriented regarding place and

Author affiliations: Canadian International Water and Energy Consultants Clinic Travel Medicine Center, Kathmandu, Nepal $(\mathrm{H}$. Murphy, P. Pandey); and Université de la Méditerranée, Marseille, France (A. Renvoisé, P. Parola, D. Raoult)

DOI: http://dx.doi.org/10.3201/eid1710.101943 time and had bilateral deafness, conjunctivitis, multiple lymphadenopathies, tender hepatosplenomegaly, bilateral rales, and a purpuric rash. The rash showed a predilection for the extremities, including palms and soles (Figure). There was no eschar. Pertinent laboratory values were the following: creatinine $2 \mathrm{mg} / \mathrm{dL}$ (baseline $0.8 \mathrm{mg} / \mathrm{dL}$ ), aspartate aminotransferase $105 \mathrm{U} / \mathrm{L}$, alkaline phosphatase $765 \mathrm{U} / \mathrm{L}$, and minimum platelet count $40,000 / \mathrm{mm}^{3}$. Chest radiograph showed bilateral interstitial infiltrates.

The patient was from New Zealand, had lived in Nepal for 30 years, and worked in wild dog protection. She reported removal of a tick 2 weeks before admission and contact with dogs, rats, ticks, fleas, and mosquitoes. She had returned from a 1-month visit to Queenstown, New Zealand, 3 months earlier and had stayed for 2 days in Thailand. She spent 1 year in Canberra, Australian Capital Territory, Australia, 12 years earlier. She was treated with oral doxycycline (100 mg $2 \times /$ d for 14 days) and showed defervescence by day 16 . She recovered slowly over 3 months but had persistent tinnitus and residual high-tone hearing loss bilaterally.

Serum samples were sent to the Unité des Rickettsies (Marseille, France) to identify the etiologic agent. Samples were tested by using a multiple-antigen immunofluorescent test (5). Antigens included those from SFG Rickettsia spp., typhus group Rickettsia spp., and Orientia tsutsugamushi. Increased immunoglobulin (Ig) $\mathrm{G}$ and $\mathrm{IgM}$ titers were observed, mainly for SFG rickettsiae (Table). Kinetics of antibodies titers showed seroconversion within 3 weeks of follow-up and 4-fold increases in IgG titers, which confirm a diagnosis of rickettsial infection. As usually observed for Rickettsia species, serologic cross-reactivity occurred, but the highest increased antibodies titers in convalescentphase serum were for $R$. honei (IgG 1,024, IgM 64) and $R$. felis (IgG 256, IgM 16) (Table).

The first serum sample negative for Rickettsia spp. was tested by real-time PCR. DNA was extracted from serum by using the QIAamp Tissue Kit (QIAGEN, Hilden, Germany), according to the manufacturer's instructions. The result of a PCR using a probe specific for SFG Rickettsia spp. (6) was positive (cycle threshold 34.3). Rickettsial DNA was detected by PCR amplifications of the outer membrane protein $\mathrm{A}(\mathrm{ompA})$ and ompB genes of Rickettsia spp. We obtained amplification products of $514 \mathrm{bp}$ and $100 \%$ similarity with the $R$. honei ompA gene (GenBank accession no. AF018075) and 603 bp with $100 \%$ similarity with the $R$. honei ompB gene (GenBank accession nos. AF123724 and AF123711) (2). We obtained 2 PCR products for 2 rickettsial genes, which showed $100 \%$ sequence similarity with $R$. honei. In addition, we detected increased antibodies titers for $R$. honei antigen

${ }^{1}$ These authors equally contributed to this article. 


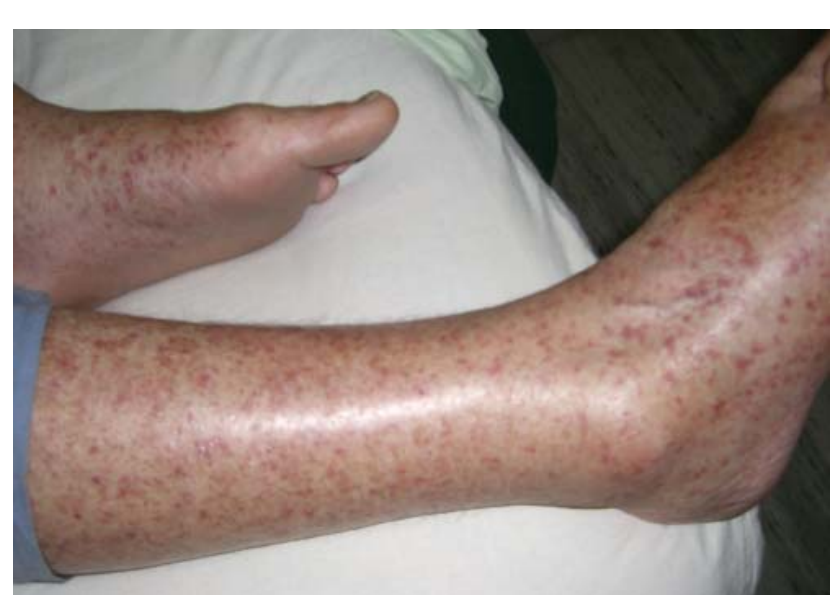

Figure. Rash exhibited by patient infected with Rickettsia honei, Nepal, 2009.

$\geq 2$-fold higher than for any other species (Table). These results and compatible clinical features confirmed the diagnosis of $R$. honei infection.

\section{Conclusions}

FISF was described in 1991 in Flinders Island (an island off the southeastern coast of Australia near Tasmania) and was similar to fever caused by an SFG rickettsia. In 1992, Rickettsia honei isolates were obtained from 2 patients with FISF. These isolates were characterized by using molecular methods and proposed as a new species in 1998 named $R$. honei $(2,3)$; strain $\mathrm{RB}^{\mathrm{T}}$ is the type strain. Thai tick typhus strain TT-118, isolated from a tick in 1962 in Thailand (2, 3 ), was shown to be a strain of $R$. honei. $R$. honei strain marmionii was detected in 2007 (7); although the precise taxonomic position of this subspecies is unknown.
$R$. honei has been associated with various tick species (3), including Ixodes granulatus (Thailand) and Haemaphysalis novaeguineae (7) (mainland Australia) associated with rats, and Aponoma hydrosauri ticks (now Bothriocroton hydrosauri) (Flinders Island, Australia) associated with reptiles. One explanation for the uncommon distribution of $R$. honei is that ectoparasites associated with migrating birds that feed on local reptiles may transmit $R$. honei to reptile ticks (3).

Human cases of $R$. honei infection have been reported on Flinders Island and elsewhere in Australia (Tasmania, South Australia, Queensland, Torres Strait Islands) since 1991 (8) and in Thailand (4). Disease occurs primarily in spring and summer and has been mild; no deaths have been reported. Common features include fever, headache, myalgia, cough, arthralgia, and maculopapular to purpuric rash without vesiculation. An eschar is reported in $50 \%$ of cases $(2,3)$.

Encephalitis, pneumonitis, tinnitus, and deafness in the patient are complications not reported with $R$. honei infections. Deafness has been reported with other SFG rickettsioses, particularly Rocky Mountain spotted fever (9) and infection with O. tsutsugamushi (10). Unsworth et al. reported 7 cases of FISF caused by $R$. honei strain marmionii that showed epidemiologic and clinical features different from those of classic FISF (7). Cases occurred in late summer and fall; cutaneous eschar was uncommon, and rash was not found on palms and soles of extremities (7). However, severe disease did not develop in any of these case-patients. The case we report differs from both patterns described. Unsworth et al. reported 1 chronic case of infection with $R$. honei strain marmionii, isolation of rickettsiae on day 27 , and 1 patient with recrudescent

\begin{tabular}{|c|c|c|c|c|}
\hline \multirow[b]{2}{*}{ Species tested } & \multicolumn{4}{|c|}{ IgG/lgM titer by date } \\
\hline & Apr 20 & Apr 24 & Apr 29 & May 15 \\
\hline R. honei & Neg & $256 / 32$ & $512 / 32$ & $1,024 / 64$ \\
\hline R. felis & $32 / 0$ & $256 / 16$ & $256 / 16$ & $256 / 16$ \\
\hline R. massiliae & Neg & Neg & Neg & $128 / 16$ \\
\hline R. aeschlimannii & Neg & Neg & Neg & $128 / 16$ \\
\hline R. conorii subsp. israelensis & Neg & Neg & Neg & $128 / 16$ \\
\hline R. conorii subsp. conorii & Neg & Neg & Neg & $128 / 16$ \\
\hline R. conorii subsp. mongolitimonae & Neg & Neg & Neg & $128 / 18$ \\
\hline R. slovaca & Neg & Neg & $\mathrm{Neg}$ & $128 / 16$ \\
\hline R. helijongangensis & Neg & $32 / 32$ & $64 / 32$ & $64 / 64$ \\
\hline R. AT1 & Neg & $32 / 32$ & $64 / 32$ & $64 / 64$ \\
\hline R. africae & Neg & $32 / 32$ & $64 / 32$ & $64 / 64$ \\
\hline R. japonica & Neg & $32 / 32$ & $64 / 32$ & $64 / 64$ \\
\hline R. conorii subsp. indica & Neg & Neg & $64 / 32$ & $64 / 32$ \\
\hline R. typhi & Neg & Neg & Neg & $64 / 64$ \\
\hline R. prowazekii & Neg & Neg & Neg & $64 / 64$ \\
\hline Orientia tsutsugamushi serotype Kawasaki & Neg & Neg & Neg & $64 / 64$ \\
\hline O. tsutsugamushi serotype Gilliam & Neg & $\mathrm{Neg}$ & $\mathrm{Neg}$ & Neg \\
\hline
\end{tabular}

${ }^{*} \mathrm{Ig}$, immunoglobulin; neg, negative. Titer cutoff values were $\geq 128$ for IgG and $\geq 64$ for IgM. A negative titer was rep
result was negative. A titer of 0 was reported when an initial screening result was positive but no Ig was detected. 
disease (day 33) after a 10-day symptom-free period (11). However, data from other studies, such as detection of $R$. honei in ticks in Texas (3) or in blood of patients without fever (8), could represent PCR contamination; these results need to be confirmed (12).

The case in this study provides evidence for human infection with SFG rickettsiosis in Nepal, where murine typhus and scrub typhus have only recently been described (1) and SFG rickettsioses have only been suspected (13). A preliminary study reported isolation of strain TT-118 from a Rhipicephalus haemaphysaloides ticks in Nepal (14). Moreover, Ix. granulatus ticks, which are associated with $R$. honei in Thailand, have been found in Nepal (15).

We propose that FISF may be an endemic rickettsiosis in Nepal and that studies of SFG Rickettsia spp., particularly $R$. honei in this region, are needed. Our report of $R$. honei infection in Nepal suggests a broader geographic distribution of FISF than believed. Clinical and entomologic research may improve our understanding of the etiology of febrile illness and the neglected field of emerging rickettsioses in Asia.

This study was supported by the French Centre National de la Recherche Scientifique.

Dr Murphy is a physician specializing in clinical infectious diseases in Kathmandu, Nepal. Her research interests are infectious diseases, public health, and tropical medicine.

\section{References}

1. Murdoch DR, Woods CW, Zimmerman MD, Dull PM, Belbase RH, Keenan AJ, et al. The etiology of febrile illness in adults presenting to Patan Hospital in Kathmandu, Nepal. Am J Trop Med Hyg. 2004;70:670-5.

2. Parola P, Paddock CD, Raoult D. Tick-borne rickettsioses around the world: emerging diseases challenging old concepts. Clin Microbiol Rev. 2005;18:719-56. doi:10.1128/CMR.18.4.719-756.2005

3. Graves S, Stenos J. Rickettsia honei: a spotted fever group Rickettsia on three continents. Ann N Y Acad Sci. 2003;990:62-6. doi:10.1111/j.1749-6632.2003.tb07338.x
4. Jiang J, Sangkasuwan V, Lerdthusnee K, Sukwit S, Chuenchitra T, Rozmajzl PJ, et al. Human infection with Rickettsia honei, Thailand. Emerg Infect Dis. 2005;11:1473-5.

5. Parola P, Miller RS, McDaniel P, Telford SR, Rolain JM, Wongsrichanalai $\mathrm{C}$, et al. Emerging rickettsioses of the Thai-Myanmar border. Emerg Infect Dis. 2003;9:592-5.

6. Socolovsch C, Mediannikov O, Sokhna C, Tall A, Diatta G, Bassene $\mathrm{H}$, et al. Rickettsia felis-associated uneruptive fever, Senegal. Emerg Infect Dis. 2010;16:1140-2.

7. Unsworth NB, Stenos J, Graves SR, Faa AG, Cox GE, Dyer JR, et al. Flinders Island spotted fever rickettsioses caused by "marmionii" strain of Rickettsia honei, eastern Australia. Emerg Infect Dis. 2007;13:566-73. doi:10.3201/eid1304.060087

8. Graves S, Stenos J. Rickettsioses in Australia. Ann N Y Acad Sci. 2009;1166:151-5. doi:10.1111/j.1749-6632.2009.04530.x

9. Steinfeld HJ, Silverstein J, Weisburger W, Rattner F. Deafness associated with Rocky Mountain spotted fever. Md Med J. 1988;37: 287-8.

10. Premaratna R, Loftis AD, Chandrasena TG, Dasch GA, de Silva HJ. Rickettsial infections and their clinical presentations in the Western Province of Sri Lanka: a hospital-based study. Int J Infect Dis. 2008;12:198-202. doi:10.1016/j.ijid.2007.06.009

11. Unsworth N, Graves S, Nguyen C, Kemp G, Graham J, Stenos J. Markers of exposure to spotted fever rickettsiae in patients with chronic illness, including fatigue, in two Australian populations. QJM. 2008;101:269-74. doi:10.1093/qjmed/hcm149

12. Raoult D. Being careful with PCR to avoid erroneous discoveries. Scand J Infect Dis. 2011;43:323-4. doi:10.3109/00365548.2011.55 4857

13. World Health Organization. Global surveillance of rickettsial diseases: memorandum from a WHO meeting. Bull World Health Organ. 1993;71:293-6.

14. Fujita H, Haithong U, Akada N. Preliminary report on rickettsial strains of spotted fever group isolated from ticks of China, Nepal and Thailand [in Japanese]. Ohara Sogo Byoin Nenpo. 2002;44: $15-8$.

15. Clifford CM, Hoogstraal H, Keirans JE. The Ixodes ticks (Acarina: Ixodidae) of Nepal. J Med Entomol. 1975;12:115-37.

Address for correspondence: Didier Raoult, Unité de Recherche en Maladies Infectieuses et Tropicales Emergentes, Centre National de la Recherche Scientifique-Institut de Recherche pour le Développement, Unite Mixte de Recherche 6236-198, Faculté de Médecine, Université de la Méditerranée, 27 Blvd Jean Moulin, 13385 Marseille CEDEX 5, France; email: didier.raoult@gmail.com

\section{Like our podcasts?}

Sign up to receive email announcements when a new podcast is available.

www.cdc.gov/ncidod/eid/subscrib.htm

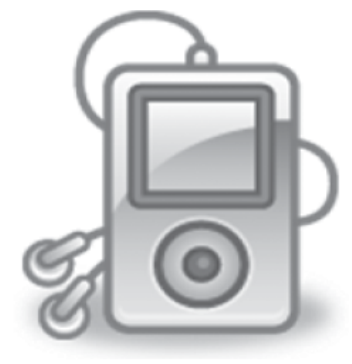

